\begin{tabular}{|c|c|c|c|c|c|}
\hline $\begin{array}{c}\text { Boletín Científico } \\
\text { CCCP. }\end{array}$ & $\begin{array}{c}\text { TUMACO-NARIÑO } \\
\text { (Colombia) }\end{array}$ & No. 3 & $3-13$ & Junio 1992 & $\begin{array}{c}\text { ISSN } \\
0121-3423\end{array}$ \\
\hline
\end{tabular}

\title{
Estudio de la influencia de la marea en el río Guapi
}

Por: Mario A. Palacios Moreno César Augusto Pinto Tovar

\section{RESUMEN}

Se determinaron los sitios hasta donde las mareas ejercen influencia en la desembocadura del río Guapi, tanto de manera directa, a través de la penetración de agua salobre, como indirecta, mediante el represamiento de sus aguas y el eventual cambio de dirección de la corriente superficial; haciendo mediación de salinidad y temperatura, y realizando observaciones sobre la vegetación predominante en las riberas y la dirección de corrientes superficiales durante los distintos niveles de marea en un período de puja que, de acuerdo a la tabla de pronósticos del HIMAT, incluía la pleamar más alta del primer semestre del año de 1992 en la Costa Pacífica Colombiana. Además se hicieron encuestas con pescadores y moradores ribereños sobre el tipo de especies ícticas capturadas en la 28 estaciones de muestreo.

\begin{abstract}
The area and importance of tidal influence on the mouth of Guapi river (Colombian Pacific Coast) was determinated. Two aspects were considered: dirct inflow of salt water and effects on the repressing of the river. In order to obtain information about these influences, were made: 1. Salinity and temperature measures up on the river. 2. Observations about the prevailing vegetation on the river's fringe and. 3. Superficial stream direction on different tide levels, including a period with the semiannual highest value of tides. Furthermore, the fishermen communities of the river border were consulted about the principal fish species catched in 28 sample stations.
\end{abstract}

\section{INTRODUCCION}

La desembocadura del rio Guapi, como todos los estuarios, se caracteriza por estar fuertemente influenciada por la acción de las mareas, cuyo rango de variación en la Costa Pacífica Colombiana es de aproximadamente $3.7 \mathrm{~m}$, ejerciéndo por consiguiente gran influencia sobre las comunidades litorales (Prahl et al., 1990).

La salinidad constituye un elemento principal en la selección y distribución de las especies que habitan en las riberas y aguas del río bajo la in- fluencia oceánica, ya que a través de las mareas se inyectan grandes cantidades de agua salobre río arriba. Sinembargo la salinidad a lo largo de la desembocadura del río, al igual que la temperatura y otros parámetros físico-quimicos del agua, está en función de múltiples factores tales como el tipo de marea (de sicigia o cuadratura), caudal del río, morfología costera, tipo de suelo, factores meteorológicos, etc.

Por lo anterior, se considera que la observación de la vegetación de la ribera del rio es la principal 
fuente de información para la tarea de estimar hasta qué punto el agua marina afecta, río arriba, la composición de la biota; ya que constituye un indicador espacio temporal adecuado.

El presente estudio pretende estimar el grado al cual se extiende la influencia de las mareas a lo largo de la desembocadura del río Guapi, por medio de mediciones de salinidad y temperatura y de observaciones de la dirección de la corriente, del tipo de vegetación ribereña y de las especies más comúnmente capturadas por los pescadores artesanales; además de encuestas con entidades locales relacionadas con usos y manejos de los suelos, pesca, agricultura y promoción social.

\section{MATERIALES Y METODOS}

\subsection{Area de Estudio}

El Municipio de Guapi, fundado en 1772 por el español Manuel de Valverde, está ubicado en la Costa Pacífica del Departamento del Cauca. Limita al Norte con el Municipio de Timbiquí; por el Sur con el Municipio de Iscuandé; por el Oriente con la Cordillera Occidental y por el Occidente con el Océano Pacífico. Está ubicado a 2034' de latitud Norte y 77056' de latitud Sur. Su superficie aproximada es de $2.688 \mathrm{~km} 2$. Según el censo poblacional de 1985 , tiene 22.166 habitantes, de los cuales 7.612 se distribuyen en el área urbana y 14.554 en la rural. Está surcado por varios ríos caudalosos que nacen en la Cordillera Occidental y que en su desembocadura crean una amplia red de esteros; entre ellos los principales son el Guapi, el Guaji y el Napi. En un principio Guapi fue un pueblo de pescadores pero actualmente la economía se sustenta en partes iguales por la minería (cabeceras de los rios Napi y Guajui principalmente), la extracción maderera, la misma pesca en altamar, pues en el río es poco rentable, debido supuestamente a los afectos negativos de la minería sobre ella, y la agricultura cultivándose, principalmente coco y maíz con algunos intentos poco fructíferos de introducir la palma africana; actualmente existe una fábrica enlatadora de Palmito, que aprovecha la palma Naidí (Euterpes Cuatrecasana) que crece silvestre (Portocarrero, Com. per.).

Las fluctuaciones de las mareas denominadas pujas y quiebras grandes y pequeñas, con duración de 7 a 8 dias de acuerdo con la mayor o menor altura, represa los rios hasta $50 \mathrm{kms}$. de su bocana; la temperatura media anual es de $26^{\circ} \mathrm{C}$, apreciándose temperatura baja en las horas de la madrugada; la precipitación promedio anual oscila entre 2.000 y $5.000 \mathrm{~mm}$; la humedad relativa es alta, casi del $100 \%$;. La altura promedio a lo largo de la Costa Caucana sobre el nivel del mar es inferior a $10 \mathrm{~m}$; la topografía es ligeramente ondulada con pequeñas depresiones y con un nivel freático super- ficial, lo cual impide la mecanización para explotación agrícola y la construcción de obras de infraestructura; el suelo es cenagoso, de color pardo negruzco, contiene alta proporción de materia orgánica y limo, especialmente en las zonas próximas a la desembocadura de los ríos; en áreas de mayor inundación, está conformado por arcilla cruda, y es arenoso en las zonas de menor influencia del agua (Villamil y Cárdenas, 1976).

\subsection{Metodología}

Se recorrió el río Guapi desde su desembocadura hasta las zonas donde la vegetación y las encuestas realizadas indicaban que no existía la más mínima influencia de la marea. El recorrido por el río se hizo en una lancha con motor fuera de borda, tanto desde la bocana del Guapi como desde las de los ríos Guajui (Brazo Limones) y del estero Quiroga, por considerarse que a través de éstos también hay penetración de aguas oceánicas que pueden influir en el cause principal del río.

Una vez hecho el reconocimiento inicial del área se efectuaron cinco jornadas de muestreo que incluyeron los siguientes recorridos: desde la bocana del río Guapi hasta la boca del Guajui (navegando paralelo a la costa), y de ésta, por el río Guajui, hasta el brazo Limones del río Guapi (este brazo desemobca en el río Guajui, justo antes de su entrada al mar); llegando luego hasta el Partidero y de allí hasta Guapi. El segundo recorrido fue desde la bocana del Guapi hasta el Partidero, pasando por Guapi y descendiendo por el brazo Limones. El tercer recorrido fue Guapi Partidero - Limones - Quiroga - Bocana de Quiroga. El cuarto recorrido fue Guapi - Partidero - Calle Honda y Caimito. Finalmente se hizo el recorrido desde bocas de Temuey hasta la Bocana del Guapi (Figura 1). El recorrido hasta Caimito se hizo durante la marea máxima del día 23 de Febrero $(4.22 \mathrm{~m})$, para precisar mejor la influencia de la marea; de igual forma se aprovechó la marea máxima del día 22, 4.28 m. (HIMAT, 1992), para el mismo efecto, durante el segundo recorrido. 


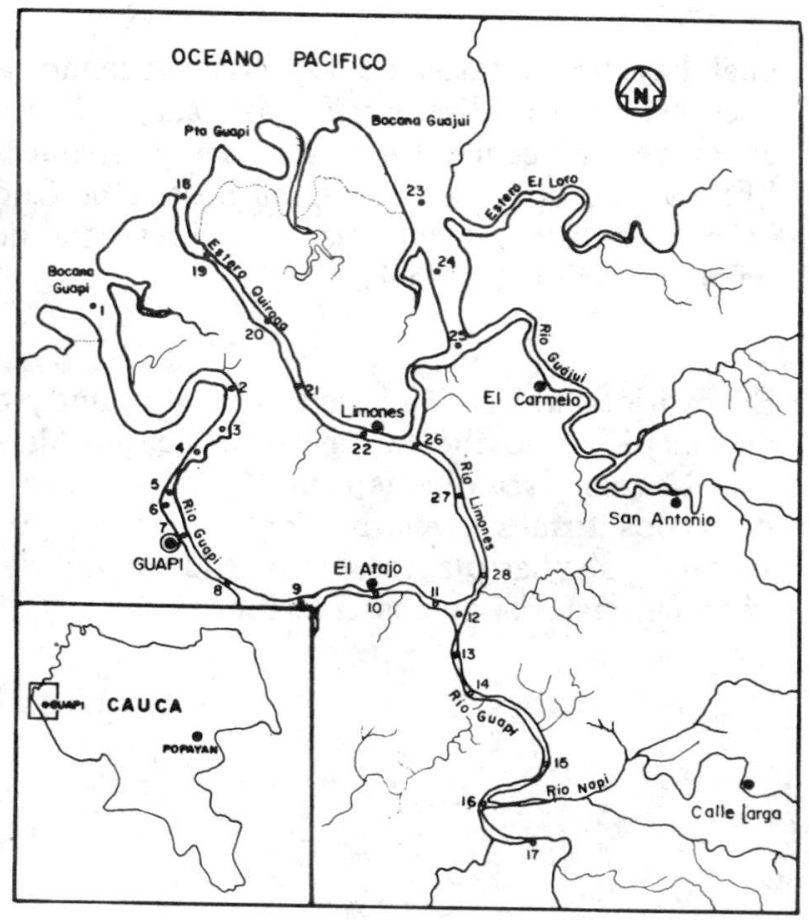

FIG. 1: Ubicación de las estaciones en el río Guapi, estero Quiroga y Brazo Limones.

En estos trayectos se hicieron muestreos y tomas de datos de temperatura con termómetro de cazoleta $0-500^{\circ} \mathrm{y}$ de salinidad con un refractómetro S-1 de $0-160 \% / o o$, tanto en fondo como en superficie; como mínimo se tomaron tres muestras en cada una de las veintiocho (28) estaciones, procurando hacerlo tanto en marea máxima como mínima; las muestras de fondo se obtuvieron con una botella Nansen, previa medición de la profundidad del lugar con un cabo metrado de $30 \mathrm{~m}$. y simultáneamente se determinaba la dirección de la corriente superficial con boyas de deriva para observar si ésta se dirigía hacia el mar o en sentido contrario.

En cada estación se encuestaron pescadores y moradores del lugar para obtener datos sobre capturas de especies acuáticas (peces, crustáceos y moluscos), tipo de vegetación y comportamiento del río con respecto a las mareas, inquiriendo además sobre los efectos de las lluvias, crecidas del río y epocas del año sobre cada uno de los aspectos descritos por ellos.

Para obtener información más precisa sobre pesca, uso de los suelos hidrografía y demás aspectos del estudio, se hicieron consultas a entidades locales como la Prefectura Apostólica; la Seccional del Instituto Colombiano de la Reforma Agraria, INCORA; Plan de Desarrollo Integral para la Costa Pacífica PLADEICOP, y la regional de la Corporación Autónoma para el Desarrollo del Cauca, CRC. En la ciudad de Cali, se visitó la Universidad del Valle, la Corporación Autónoma Regional para el desarrollo del Valle del Cauca (CVC), PLADEICOP y el INCORA.

\section{RESULTADOS}

La profundidad en el río Guapi, estero Quiroga y Brazo Limones es muy variable porque depende en gran medida de la marea y del caudal del río que aumenta en época lluviosas y disminuye en las secas. Sinembargo, se puede decir que se encontraron profundidades mínimas en la boca del estereo Quiroga de $3.5 \mathrm{~m}$ y máximas en frente al islote antes de llegar a Guapi de $10 \mathrm{~m}$.

En general, la salinidad y temperatura superficial son menores que las del fondo. En la Tabla 1 se presentan los valores medios de estos dos parámetros por estación y la ubicación geográfica de las mismas.

Los resultados de las encuestas dejaron en claro los siguientes puntos:

- Durante las épocas de pujas, la marea sube hasta el Cacerio de Caimito por el río Guapi y hasta el de Playa del Medio sobre el río Napi.

- Cuando hay época seca, principalmente en las cabeceras de los ríos, en períodos de puja el agua alcanza a ponerse salobre frente al municipio de Guapi. Por el contrario, desde el Partidero hacia arriba (río arriba), nunca ocurre ésto.

- En el embarcadero de Guapi se pesca "Canchimalo" Arius multirradiatus, aunque también existe un reporte de pesca de esta misma especie en Temuey, y desde Chamoncito hacia el mar, principalmente desde Cantil, se pesca la Jaiba Callinectes toxotes, Bagre Bagre spp. Gualajo Centropomus spp. y Ñato Arius spp.

- Sobre el Brazo Limones se pesca Jaiba y Bagre hasta el corregimiento de Limones, de este lugar en adelante (río arriba) desaparecen estas especies, aunque en las Pampas reportaron capturas eventuales de Bagre y Canchimalo.

Las observaciones sobre la vegetación arrojaron los siguientes resultados:

- En la Bocana de Guapi predominan el Mangle Rojo Rhizophora spp. y el Mangle Negro Avicen- 
nia germinans; lo mismo se observa en las bocanas de Quiroga y Guajui (Figura 2). Sobre el río Guapi, desde la salida del canal artificial (construido por Caminos Vecinales), se termina la presencia del $R$. spp. y comienza el predominio de $A$. germinans (Figura 3 ). Adicionalmente, dentro de este canal artificial de márgenes muy intervenidas, se observa la presencia de Nato Mora megistosperma, del helecho conocido como Ranconcha Acrostichium aureum y palmas de Coco.

- A partir de Chamoncito, comienza a desaparecer el Mangle Negro especialmente en la margen sur del río. Es después del Islote, frente al cual hay una estatua de la Virgen, cuando se hace evidente la disminución del Mangle Negro en ambos márgenes. En terreros de la pesquera "Frutos del Mar" se observa un bosque de Sajo Camnosperma panamensis y la presencia de palma de Naidi (Figura 4).

- En el islote ubicado a la entrada del municipio de Guapi, río arriba, se observan algunos Mangles Negros. Las riberas a partir del islote son de suelos firmes y relativamente elevadas, presentando algunas playas de donde los moradores obtienen material de construcción.

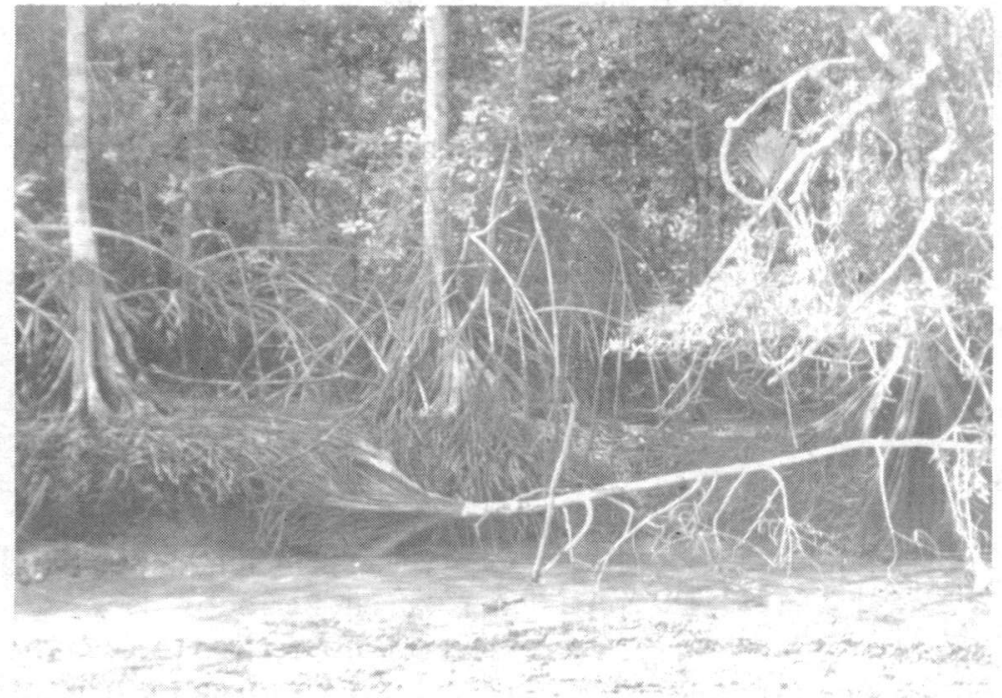

FIG. 2

Mangle rojo y negro en la Bocana de Guajui, tornado con marea baja.

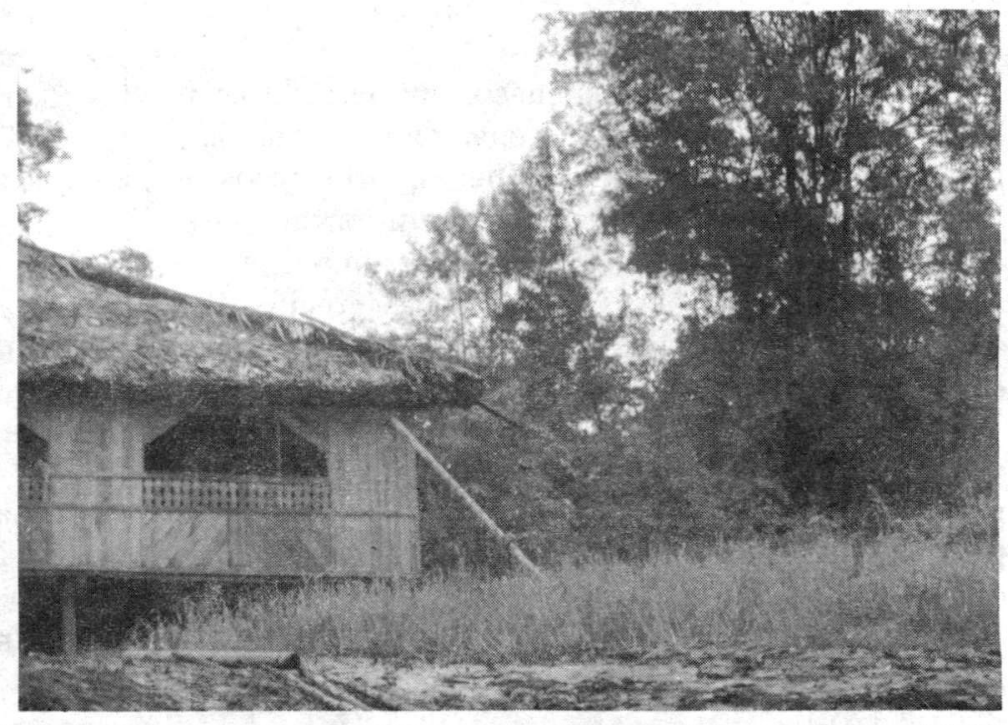


FIG. 4 :

Arboles de Sajo y Palmas Naidi adelante de Chamoncito río Guapi arriba.

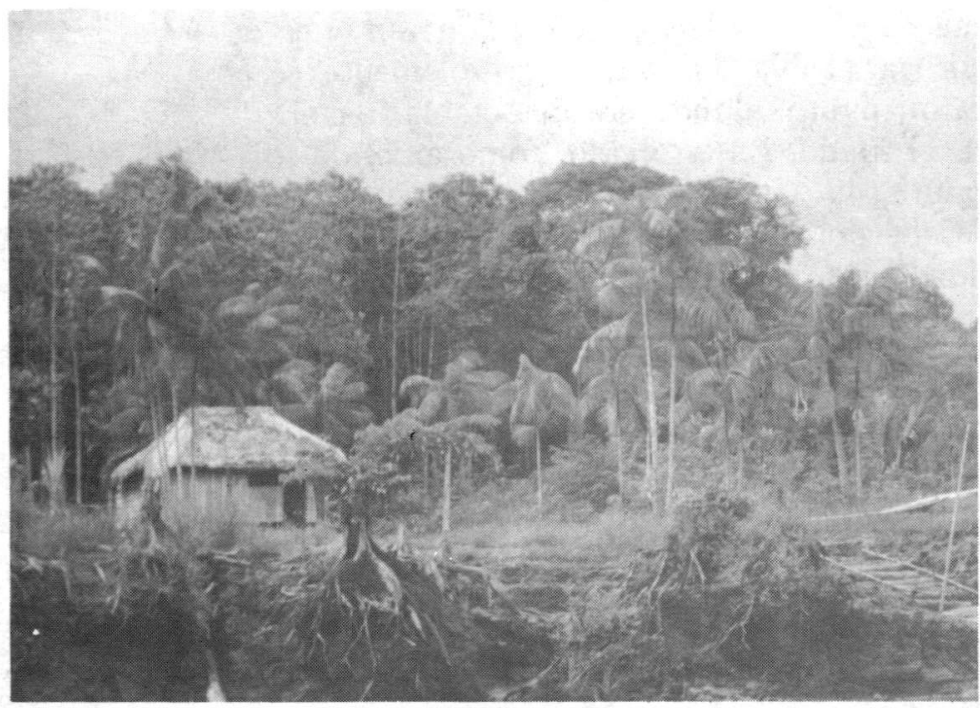

- La bocana de Quiroga muestra a su entrada la presencia de Mangle Rojo que se alterna con Mangle Negro aproximadamente hasta la altura de la primera plantación de coco, en el estero de Quiroga hay alrededor de cinco plantaciones de coco entre los bosques ribereños de $A$. germinans. El Mangle Negro predomina a partir de allí (Figura 5) y desaparece casi por completo en la estación de Juanico; sinembargo, desde la central de acopio de Coco, éste ya empieza a alternar con Nato y a partir de los caseríos de Quiroga y Limones con Naidí (Figura 6).

- En Pampas, antes de Partidero, se encuentra M. megistosperma, Cuangare Virola sp. y Guabo. A partir del Partidero desaparece el Nato.

- En la estación de Bonanza sobre el río Guapi ya no se observa el Nato, la vegetación predominante está constituida por Cuangare y Sajo. Frente a una planta procesadora de coco, se vuelve a apreciar la presencia de Nato, especialmente en la ribera de una pequeña quebrada que desemboca al río.

- Después de Partidero río arriba no se observa vegetación halófita, ni siquiera Nato (Figuras 7 y 8$)$.

El lanzamiento de las boyas de deriva permitió establecer que, efectivamente, con marea máxima y en época de puja, el agua del río Guapi se represa y cambia de dirección (río arriba), hasta la localidad de Caimito.

\section{DISCUSION DE RESULTADOS}

\section{SALINIDAD Y TEMPERATURA}

Tal como lo manifiestan Prahl et. al (1990), la definición de estuario es perfectamente aplicable a la mayoría de bahías, golfos, desembocaduras de ríos y lagunas costeras de la Costa Pacífica Colombiana ya que son cuerpos de agua semiencerrados con libre conexión al mar abierto y en los cuales se producen mezclas periódicas de agua dulce y agua salada. Esta mezcla de aguas no es estable, sino que presenta cambios periódicos (Casper, 1976 en Prahl et al 1990). En la Costa Pacífica estos cambios se deben principalmente a las corrientes de convección que se generan por los flujos de las mareas y la poca profundidad de los estuarios (Pathl et. al., 1990).

En las Figuras 9, 10 y 11 se observa la variabilidad de las mezclas de agua salada y dulce y de la temperatura a lo largo de la desembocadura del río Guapi y de las dos bocas conexas (Quiroga y Guajui). Se puede apreciar en primer término que la temperatura superficial generalmente es mayor que la del fondo sin superar, esta diferencia, el valor de 01 grados centígrados en cada estación. Mientras que, la variación a lo largo del río en sentido Bocana Cabecera es mayor (Máxima de 28.50 y Mínima 25.45 grados centígrados) (Tabla 1) disminuyendo en este sentido. En cuanto a la salinidad el caso es muy similar, ya que la de fondo es mayor que la superficial, aunque aqui en algunos sectores se aprecian diferencias significativas (más de 08 ppm) como en los casos de 
la Bocana de Guapi y la Quebrada Trina en el estero Quiroga (Tabla 1), donde posiblemente se muestrearon puntos donde era detectable la cuña salina en el fondo. En este caso, como en el de la Temperatura, la salinidad disminuye en sentido Bocana Cabecera. En general, se puede decir que la salinidad es Cero $(0 \%$ oo $)$ a partir del Partidero (rio arriba).

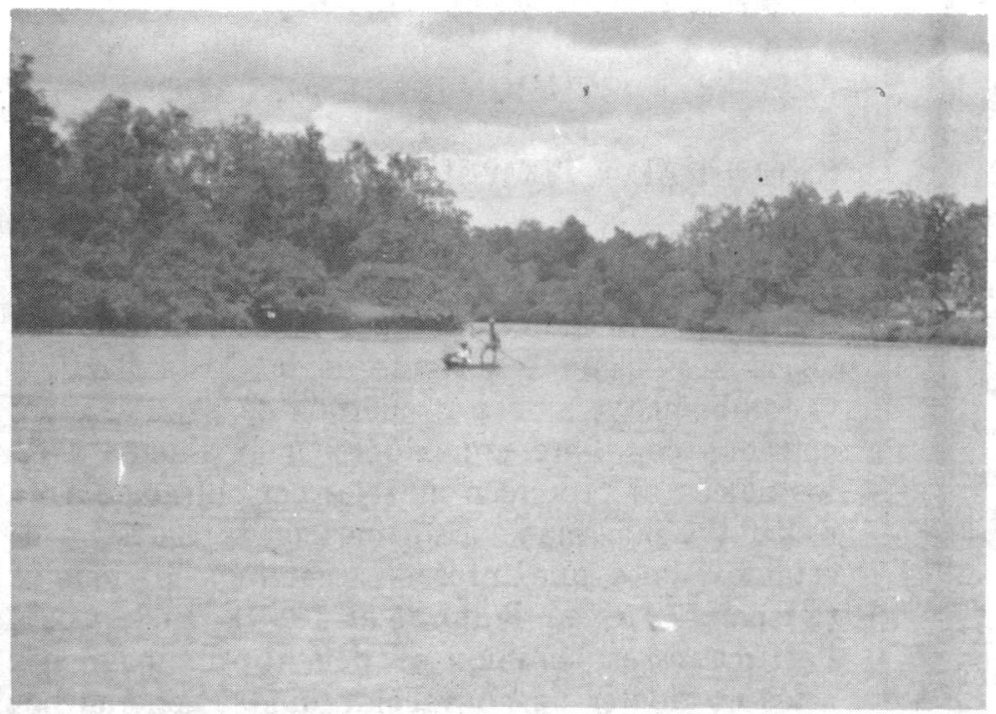

FIG. 5: Islote en el estero Quiroga donde predomina el Mangle Negro alternando con Palmas de Coco.

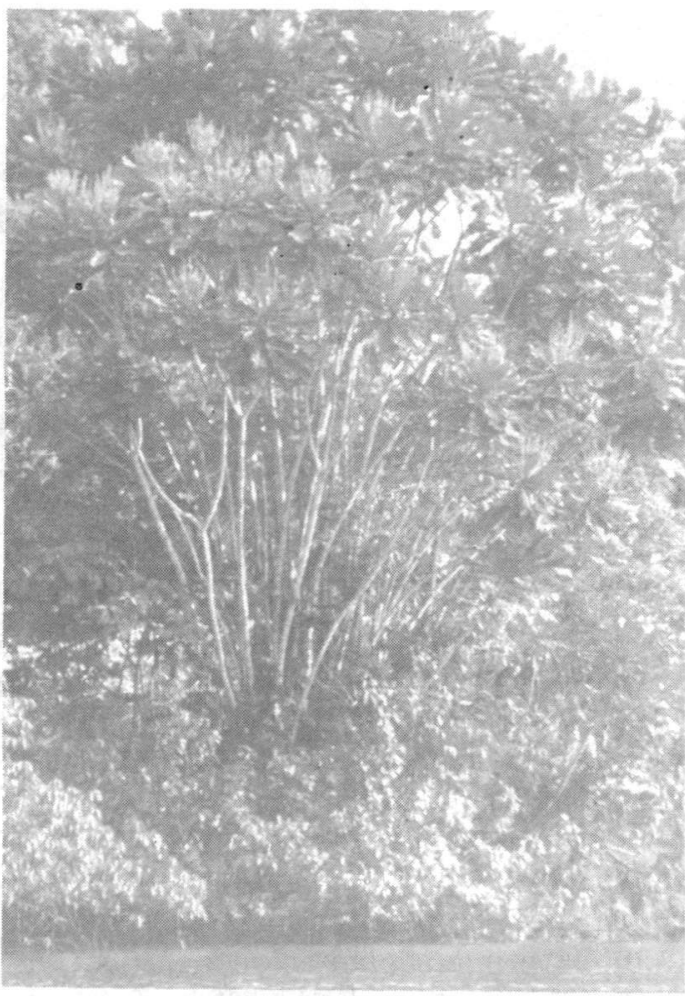

FIG. 7: Vegetación en la ribera río arriba adelante del Partidero. El árbol en flor es conocido como "Tuna". 
FIG. 8:

Vegetación predominante en la estación de Caimito.

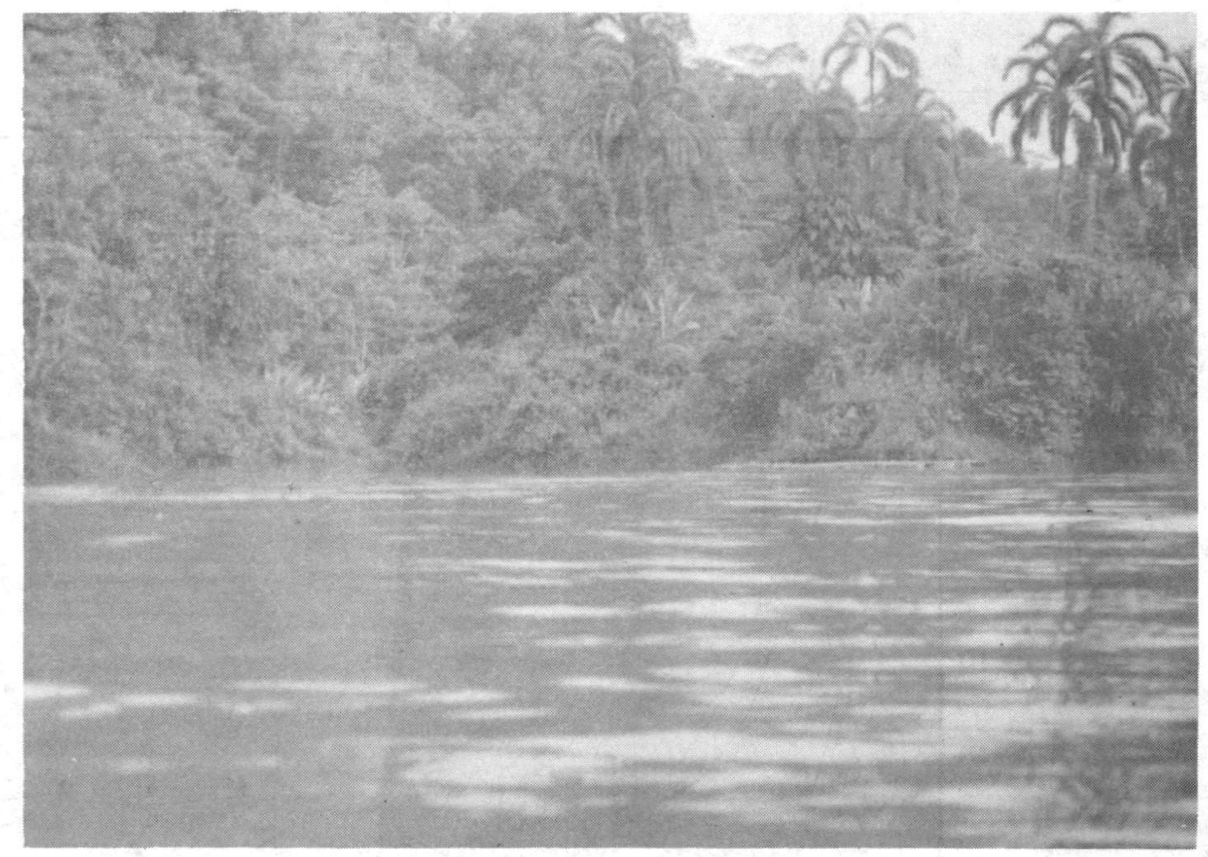

TABLA No. 1: Valores medios de Salinidad y Temperatura en superficie y fondo de posición de las Estaciones sobre el río Guapi.

\begin{tabular}{|c|c|c|c|c|c|c|c|}
\hline & \multirow{2}{*}{ ESTACION } & \multicolumn{2}{|c|}{ SALINIDAD } & \multicolumn{2}{|c|}{ TEMPERATURA } & \multicolumn{2}{|c|}{ POSICION } \\
\hline & & SUPERF & FONOO & SUPERF & FONDO & $w$ & $\mathrm{~N}$ \\
\hline 1 & Bocano Guopi & 17.00 & 25.60 & 27.80 & 28.00 & $77^{\circ} 57^{\prime} 43^{\prime \prime}$ & $2^{\circ} 37^{\prime} 32^{\prime \prime}$ \\
\hline 2 & Contil & 6.25 & 10.25 & 27.50 & 27.95 & $55^{\prime} 33^{\prime \prime}$ & $37^{\prime} 32^{\prime \prime}$ \\
\hline 3 & Chomon & 2.40 & 3.16 & 27.43 & 27.27 & $55^{\prime} 15^{\prime \prime}$ & $36^{\prime} 05^{\prime \prime}$ \\
\hline 4 & Chamoncito & 1.33 & 2.00 & 26.70 & 26.70 & $55^{\prime} 45^{\prime \prime}$ & $35^{\prime} 36^{\prime \prime}$ \\
\hline 5 & Sabana & 1.00 & 1.00 & 26.43 & 26.70 & $56^{\prime} 12^{\prime \prime}$ & $34^{\prime} 11^{\prime \prime}$ \\
\hline 6 & Islote (virgen) & 0.50 & 0.50 & 26.55 & 26.55 & $56^{\prime} 12^{\prime \prime}$ & $34^{\prime} 46^{\prime \prime}$ \\
\hline 7 & Guopi & 0.25 & 0.40 & 26.28 & 26.34 & $56^{\prime} 12^{\prime \prime}$ & $33^{\prime} 46^{\prime \prime}$ \\
\hline 8 & Bonanzo & 0.31 & 0.22 & 26.54 & 26.41 & $54^{\prime} 16^{\prime \prime}$ & $32^{\prime} 28^{\prime \prime}$ \\
\hline 9 & Temuey & 0.20 & 0.06 & 26.08 & 26.36 & $53^{\prime} 18^{\prime \prime}$ & $32^{\prime} 28^{\prime \prime}$ \\
\hline 10 & Procesadora Coco & 0.57 & 0.08 & 25.76 & 25.82 & $51^{\prime} 40^{\prime \prime}$ & $33^{\prime} 01 "$ \\
\hline 11 & Aserrio Partidero & 0.50 & 0.50 & 25.63 & 25.65 & $50^{\prime} 30^{\prime \prime}$ & $32^{\prime} 48^{\prime \prime}$ \\
\hline 12 & Partidero & 0.29 & 0.29 & 25.49 & 25.66 & $49^{\prime} 57^{\prime \prime}$ & $31^{\prime} 30^{\prime \prime}$ \\
\hline 13 & I km arribo Parlidero & 0.00 & 0.00 & 25.90 & 26.00 & $49^{\prime} 57^{\prime \prime}$ & $31^{\prime} 43^{\prime \prime}$ \\
\hline 14 & Curvo Partidero & 0.00 & 0.00 & 25.90 & 25.45 & $48^{\prime} 27^{\prime \prime}$ & $30^{\prime} 51^{\prime \prime}$ \\
\hline 15 & Calle Honda & 0.00 & 0.00 & 25.80 & 25.90 & $47^{\prime} 48^{\prime \prime}$ & $29^{\prime} 40^{\prime \prime}$ \\
\hline 16 & Boco del Napl & 0.00 & 0.00 & 25.90 & 25.90 & $49^{\prime} 05^{\prime \prime}$ & $28^{\prime} 23^{\prime \prime}$ \\
\hline 17 & Caimito & 0.00 & 0.00 & 25.70 & 25.85 & $47^{\prime} 35^{\prime \prime}$ & $26^{\prime} 59^{\prime \prime}$ \\
\hline 18 & Bocana Ouiroga & 22. 50 & 24.00 & 28.01 & 27.93 & $56^{\prime} 38^{\prime \prime}$ & $40^{\prime} 14^{\prime \prime}$ \\
\hline 19 & Quebrada La Trina & 13.66 & 26.50 & 28.00 & 28.00 & $55^{\prime} 46^{\prime \prime}$ & 39'09" \\
\hline 20 & Plantacion de Coco & 10.33 & 10,33 & 27.63 & 27.60 & $54^{\prime} 16^{\prime \prime}$ & $37^{\prime} 58^{\prime \prime}$ \\
\hline 21 & Centro acopio coco & 8.33 & 7.33 & 27.10 & 27.47 & $53^{\prime} 37^{\prime \prime}$ & $36^{\prime} 40^{\prime \prime}$ \\
\hline 22 & Coserio Quiroga & 5.25 & 7.25 & 26.70 & 26.90 & $51^{\prime} 54^{\prime \prime}$ & $36^{\prime} 02^{\prime \prime}$ \\
\hline 23 & Bocono Guajui & 25.50 & 22.00 & 28.50 & 2840 & $51^{\prime} 54^{\prime \prime}$ & $40^{\prime} 46^{\prime \prime}$ \\
\hline 24 & Arriba Boca Guajui & 8.00 & 950 & 27.95 & 28.15 & $51^{\prime} 15^{\prime \prime}$ & $39^{\prime} 28^{\prime \prime}$ \\
\hline 25 & Union Guajui-Limones & 1.00 & 100 & 26.20 & 26.80 & $50^{\prime} 49^{\prime \prime}$ & $38^{\prime} 11 "$ \\
\hline 26 & Limones & 1.75 & 2.33 & 25.90 & 25.97 & $51^{\prime} 15^{\prime \prime}$ & $36^{\prime} 02^{\prime \prime}$ \\
\hline 27 & Juanico & 1.50 & 1.25 & 25.68 & 25.75 & $50^{\prime} 36^{\prime \prime}$ & $34^{\prime} 31^{\prime \prime}$ \\
\hline 28 & Pampas & 0.40 & 0.50 & 25.42 & 25.50 & $49^{\prime} 18^{\prime \prime}$ & $33^{\prime} 13^{\prime \prime}$ \\
\hline
\end{tabular}




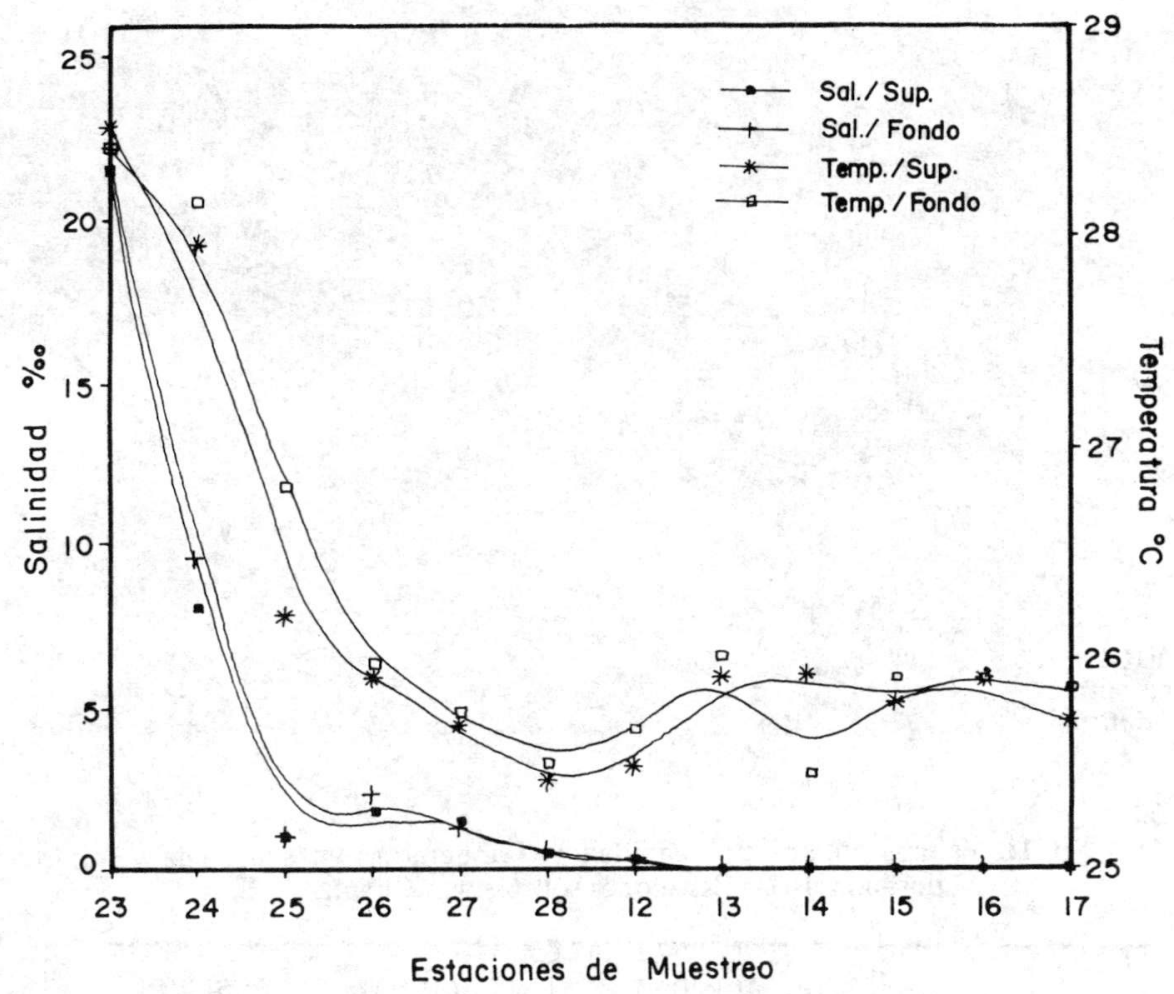

FIG. 9: Variación de Salinidad y Temperatura desde la Bocana de Guajui hasta Caimito.

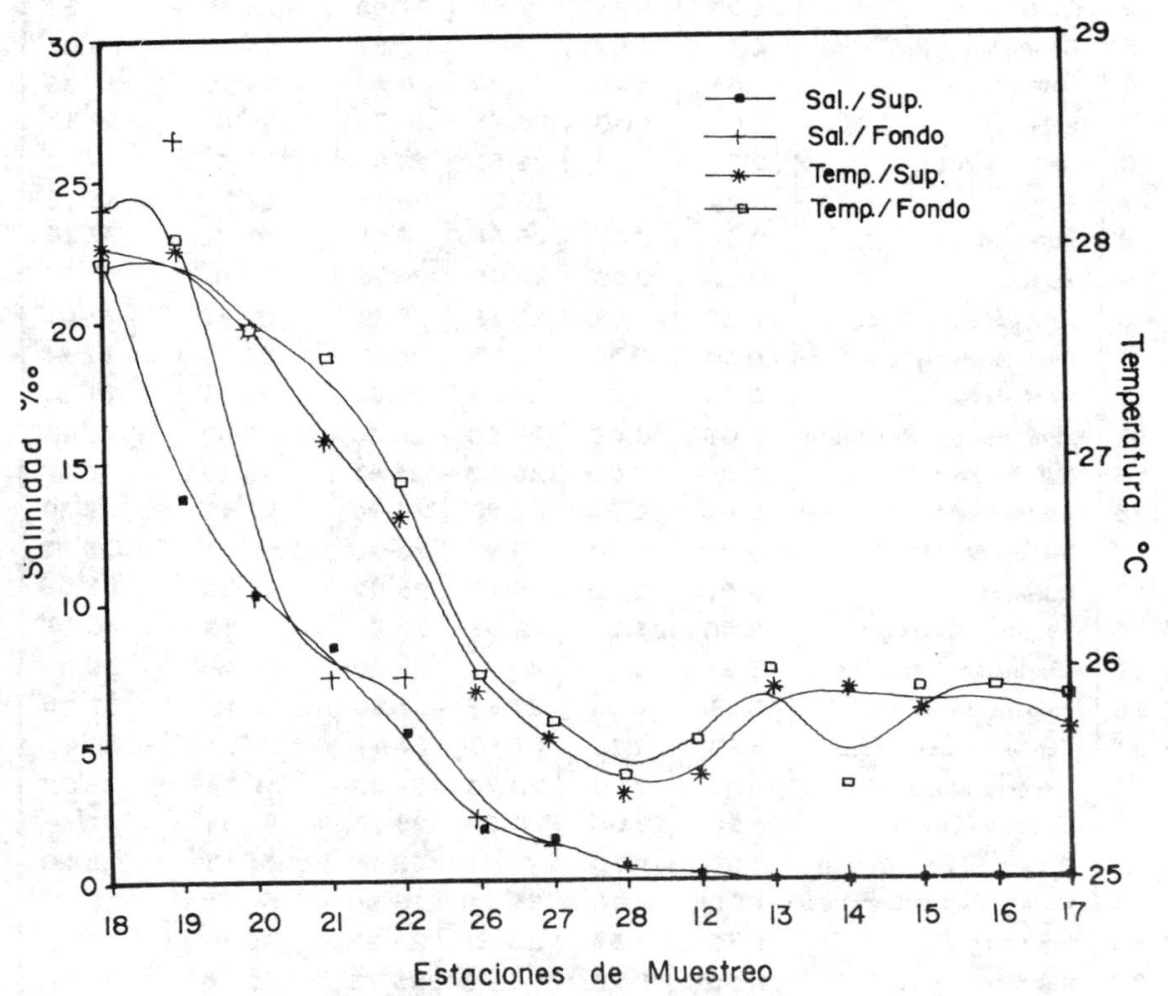

FIG. 10: Variación de Salinidad y Temperatura desde la Bocana de Quiroga hasta Caimito. 


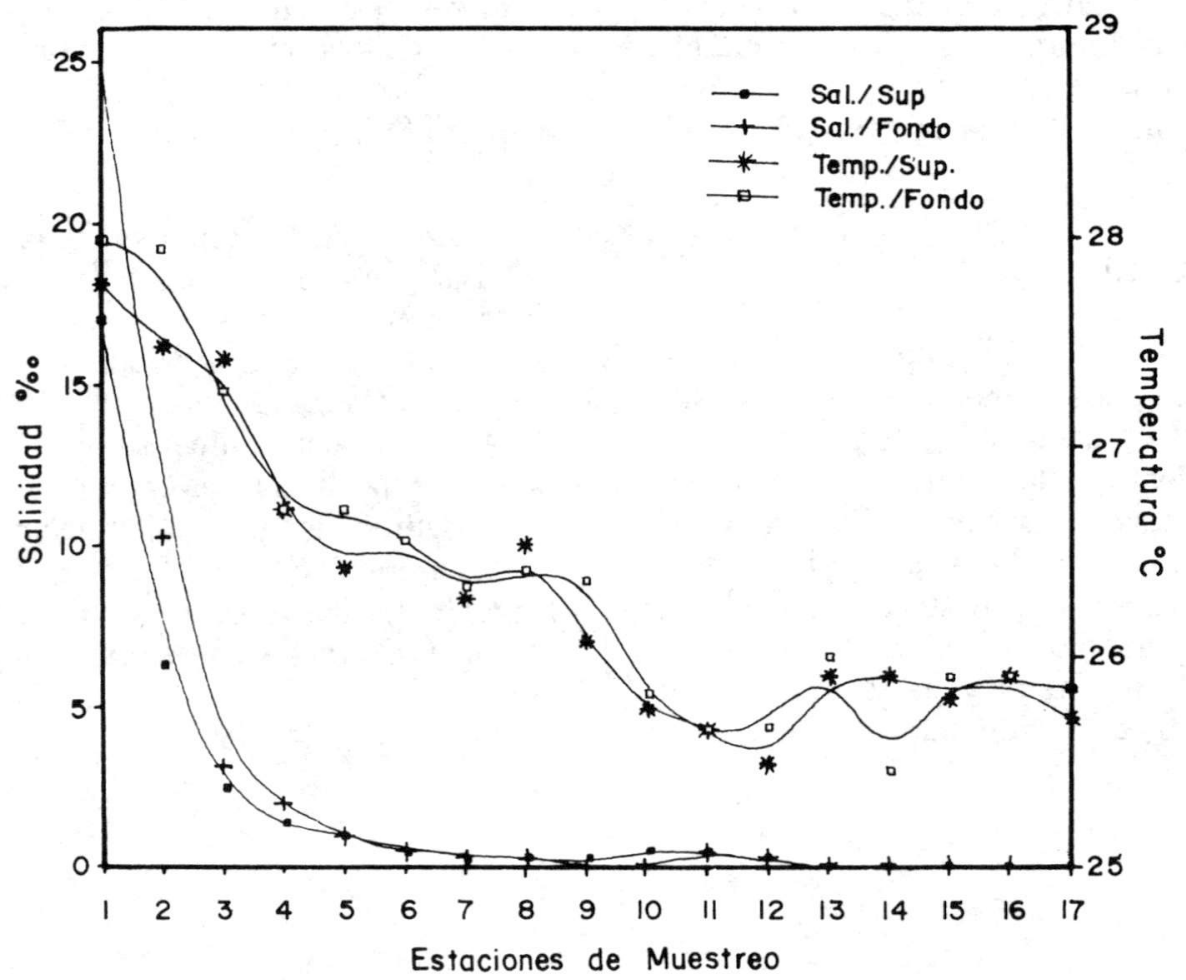

FIG 11: Variación de Salinidad y Temperatura desde la Bocana de Guapi hasta Caimito.

\section{PESCA}

Las especies marinas de interés comercial que penetran al río y sus bocanas (Bagre, Nato, Canchimala, Gualajo y Jaiba) son preferentemente habitantes de zonas estuarinas y bahías interiores donde ocupan los fondos arenosos y fangosos; pudiendo penetrar en aguas completamente dulces (Rubio et. al., 1987). Sinembargo la pesca comercial y artesanal no se realiza en el río sino que tiene lugar en el mar, salvo la Jaiba que se captura hasta Cantil en el río Guapi y hasta Juanico después de la Unión entre el estero Quiroga y el brazo Limones.

\section{VEGETACION}

La predominancia del Mangle Negro Avicennia germinans, salvo en las bocanas donde se alterna con Mangle Rojo y el terreno es más inestable, nos indica claramente que en general el sustrato ribereño objeto del estudio es consolidado y estable, ya que, como lo indica Prahl et. al. (1990), los mangles negro y blanco requieren de fondos menos inestables que el manble rojo Rhizophora spp. Los mismos autores también afirman que los mangles piñuelo y nato, sólo pueden ocupar efectivamente sustratos estabilizados; por lo cual la presencia de nato bién desarrollado a partir de la Unión del Brazo Limones con el río Guajui y desde Quiroga hasta Juanico y Pampas nos indica la ocurrencia de tal característica en este sector. Sobre el río Guapi la cantidad de Nato es mucho menor, tal vez por sobre-explotación, pero su presencia en algunas estaciones como la de la procesadora de coco nos demuestran la firmeza del sustrato de sus riberas. Además a partir de la pesquera frutos del mar, se observan árboles de sajo y cuangare, que indican con mayor claridad este hecho.

\section{CORRIENTES}

La dirección de la corriente durante las pleamares es hacia la cabecera de los ríos y su velocidad, a pesar de que no se midió, muy variable, lo cual se explica por las diferencias en la topografia submarina. En general la velocidad de estas corrientes es baja y su valor podria coincidir con el registrado por Prahl et. al (1990) de $0.5 \mathrm{~m} / \mathrm{seg}$. y que en zonas profundas puede alcanzar veloci dades de 1 y $2 \mathrm{~m} / \mathrm{seg}$. El represamiento de las aguas del río y el cambio de la dirección de su corriente superficial, se produce hasta Caimito (estación 17) y, según encuesta, hasta Playa del 
medio sobre el río Napi. Siembargo este avance es variable ya que depende de la altura de marea, del caudal del rio, que a su vez depende de la pluviosidad en su cuenca y de la morfología de su cauce.

\section{CONCLUSIONES}

Teniendo en cuenta principalmente el tipo de vegetación ribereña y los demás factores, con sus respectivas limitantes: salinidad, temperatura, di rección de la corriente, y los resultados de las encuestas sobre capturas de especies acuáticas en las distintas estaciones, se concluye que la influencia directa de las mareas a través de sus aguas salobres, se extiende hasta Juanico (estación 27) sobre el brazo Limones del rio Guapi y hasta el Islote de la Virgen (estación 6) en el río Guapi.
El represamiento de las aguas del río y eventual cambio de dirección de su corriente, efecto indirecto del flujo de mareas, por el contrario se extiende hasta Caimito (estación 17).

El efecto de la marea sobre la pesca es difícil de precisar pues, como se observa por los resultados de la encuesta, las especies de peces comerciales de origen marino, que ocasionalmente se capturan en el rio, son eurihalinos y por consiguiente pueden penetrar en agua dulce sin ningún problema. Sinembargo la Jaiba, crustáceo menos tolerante a bajas salinidades penetra únicamente hasta Juanico (estación 27) y Sabana (estación 05). Además, a partir del Partidero (estación 12), no hay reportes de captura de bagre, canchimala, Nato o Gualajo. 


\section{Bibliografía}

HIMAT, 1992. Pronóstico de pleamares y bajamares en la Costa Pacífica Colombiana, 1992.

PRAHL H. VON, J. CANTERA Y R. CONTRERAS, 1990. Manglares y Hombres del Pacífico Colombiano. Editorial Presencia.

RUBiO E., B. GUTIERREZ, R. FRANKE, 1987. Peces de la Isla de Gorgona. Centro de Publicaciones de la Facultad de Ciencias de la Universidad del Valle.

VILLAMIL, V. y H. CARDENAS. 1976. Cultivo experimental del camarón blanco en estanque. Sem. Ocea. Pac. Sud. Septiembre 1-5 pag. $62-94$ 